\section{Does delay in referral of proliferative diabetic retinopathy from the diabetic eye screening programme lead to visual loss?}

GS Negretti, R Amin, L Webster and CA Egan

\begin{abstract}
Aims To ascertain the effect on visual acuity (VA) of a delay in Hospital Eye Service (HES) consultation for patients referred with proliferative diabetic retinopathy (PDR; R3) from the Diabetic Eye Screening Programme (DESP).

Methods All patients referred to Moorfields Eye Hospital from DESP between April and December 2013 with a referral diagnosis of PDR in at least one eye were eligible. Screening programme VA was compared with VA at first HES appointment and final follow-up appointment. Reasons for any VA loss were noted.

Results A total of 86 patients were included. Of these, $28(33 \%)$ were seen in more than 4 weeks after their DESP referral. At first HES appointment, $39(45 \%)$ patients were graded as having active PDR in at least one eye. Delay in referral did not significantly predict the likelihood of vision loss in all patients referred $\left(\chi^{2}, P=0.49\right)$ or in just those patients with a definitive HES diagnosis of active PDR $\chi^{2}$, $P=1.00)$. In only 3 patients with active PDR was a delay in presentation thought to have

Introduction

In 2006, the National Diabetic Eye Screening Programme (DESP) was implemented in England (NHS DESP). The programme invites every diabetic patient over 12 years of age registered with a GP in England for screening annually. Each patient has two mydriatic digital photographic fields of each eye, graded by a quality assured team. Between 2011 and 2012, 236200 people were offered screening for diabetic retinopathy and 1911000 received screening, an uptake of $81 \%{ }^{1}$

The NHS DESP and the Hospital Eye Services (HES) managing patients referred from DESP are required to adhere to strict standards. ${ }^{1}$ One standard is that $60 \%$ of R3 grade (proliferative diabetic retinopathy (PDR)) patients referred from the DESP should receive a HES consultation within 2 weeks and $80 \%$ should receive a consultation within 4 weeks. The aim of this study was to ascertain the effect on visual acuity (VA) of a delay in HES consultation for patients referred with PDR compared with the VA at the screening visit.
\end{abstract} led directly to VA loss.

Conclusions There may be minimal shortterm visual consequence in several weeks of delayed referral for many patients with a diagnosis of R3. However, the national guidance remains important. This is due to the occasional patient at very high risk of vision loss and the many gains for the patients in terms of time to properly assess medical and ocular conditions and counsel and consent them for treatment where necessary.

Eye (2016) 30, 873-876; doi:10.1038/eye.2016.56; published online 8 April 2016

\section{Materials and methods}

This was a single-centre retrospective cohort study. Patients who were eligible for inclusion in the study were all those patients referred to Moorfields Eye Hospital (MEH) from the DESP between April and December 2013 with a referral diagnosis of PDR (R3) in at least one eye.

All patients were seen in the HES at MEH after referral from DESP. All patients had VA performed as part of a routine ophthalmology assessment. Glycated haemoglobin (HbA1C) was measured for all patients without a recent
Moorfields Eye Hospital NHS Foundation Trust, London, UK

Correspondence: GS Negretti, Moorfields Eye Hospital NHS Foundation Trust, 162 City Road, London EC1V 2PD, UK Tel: +44 (0)20 7253 3411; Fax: +44 (0)20 75214679 . E-mail: guynegretti@gmail. com

Received: 1 October 2015 Accepted in revised form: 2 February 2016 Published online: 8 April 2016 
measurement performed by their physician team and were offered testing in the context of their ophthalmology visit by the MEH diabetes specialist nurse team. They then underwent a dilated fundus exam performed by an ophthalmologist. Clinical grading of diabetic retinopathy was based on ETDRS grades and also recorded in the same way as the grading performed by the DESP ${ }^{1,2}$ for the purposes of audit and quality assurance within DESP. Wide-field fundus imaging, fundus fluorescein angiography (FFA), and optical coherence tomography (OCT) were performed where necessary to aid in diagnosis or plan treatment, but was not mandatory.

Where new vessels (NVs) were present on fundus examination, ETDRS standards were used to decide whether this represented active PDR and whether pan-retinal photocoagulation (PRP) laser was required. ${ }^{3}$

VA data at the time of screening and referral dates were provided by the DESP. Where there was any loss of vision between screening and first HES appointment or between first HES and most recent follow-up appointment, the hospital notes were used to identify the cause for this loss of vision. If the cause for the loss of the vision was a complication of PDR that could have been prevented if the patient had been seen without delay, for example, vitreous haemorrhage, neovascular glaucoma, or tractional retinal detachment, this was noted. The classification for blindness, severe visual loss, and moderate visual loss was: $<1.3$ LogMAR, 0.8-1.0

LogMAR, and 0.5-0.78 LogMAR, respectively.

Study exclusions were:

(1) Those patients referred by the DESP already under the care of $\mathrm{MEH}$ for diabetic retinopathy with active treatment plans or follow-up arranged.

(2) Those patients who did not attend any hospital outpatient appointment after their DESP referral.

This study was registered with the trust audit office, code: CA14/MR/60.

\section{Results}

A total of 167 patients were identified as eligible for the study. The screening programmes from which they were referred are shown in Figure 1.

Of these patients, 77 were excluded: 47 (61\%) patients were already under the care of $\mathrm{MEH}$ for diabetic retinopathy and 30 (39\%) did not attend any HES appointment after their DESP referral. In addition, 4 sets of notes were missing and our electronic record at the time did not contain sufficient information about these patients, leaving 86 patients for analysis. Of these patients, the average age at referral was 54 years.

There were 55 males and 31 females.

Of the patients, $27(31 \%)$ patients were seen within 2 weeks of their screening appointment; 31 (36\%) patients were seen in more than 2 weeks but less than 4 weeks; and $28(33 \%)$ patients were seen in more than 4 weeks. Of these 28 patients, 9 (32\%) were actually offered appointments within 4 weeks but failed to attend these.

The average age of those attending later than 4 weeks was 56 years compared with 53 years in those attending earlier than 4 weeks. In all, 22 males and 4 females attended later than 4 weeks. This was compared with 33 males and 25 females attending in less than 4 weeks. This gender difference had borderline significance $\left(\chi^{2}, P=0.05\right)$.

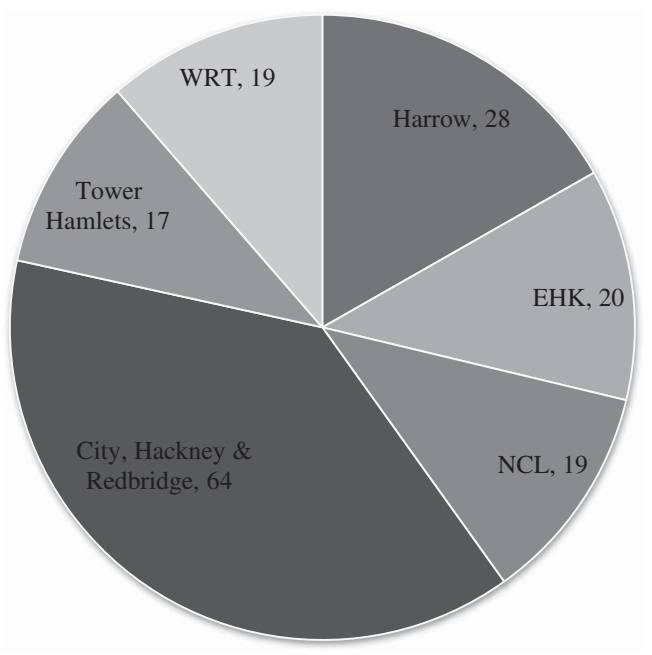

Figure 1 Number of referred patients from each screening programme. Abbreviations: NCL, North Central London; EHK, Ealing, Hounslow, and Kingston; WRT, Wandsworth, Richmond, and Twickenham.

\begin{tabular}{|l|l|l|}
\hline DR Grade & $\begin{array}{l}\text { Number of eyes } \\
\text { at Screening }\end{array}$ & $\begin{array}{l}\text { Number of eyes at first } \\
\text { HES appointment }\end{array}$ \\
\hline R3M0 & 50 & 60 \\
\hline R3M1 & 65 & 33 \\
\hline R3MU & 7 & 1 \\
\hline R2M0 & 5 & 21 \\
\hline R2M1 & 20 & 25 \\
\hline R2MU & 1 & 0 \\
\hline R1M0 & 9 & 11 \\
\hline R1M1 & 5 & 3 \\
\hline R1MU & 0 & 0 \\
\hline R0M0 & 5 & 11 \\
\hline R0MU & 0 & 1 \\
\hline RUMU & 4 & 5 \\
\hline Prosthesis & 1 & 1 \\
\hline
\end{tabular}

Figure 2 Diabetic retinopathy (DR) grade at screening and at first Hospital Eye Service (HES) visit. 
A total of 82 eyes ( $47 \%$ ) had the same grading at their first HES appointment as at screening. Screening grades and grades at first HES are shown in Figure 2. At first HES appointment, 39 (45\%) patients were graded as having active PDR in at least one eye. Of these patients, $16(41 \%)$ were seen within 2 weeks, $16(41 \%)$ in more than 2 weeks but less than 4 weeks, and $7(18 \%)$ in more than 4 weeks. Of these 7 patients, 4 (57\%) were actually offered appointments within 4 weeks but failed to attend these.

At screening, one patient was classified as blind in the better eye, two patients had severe visual loss, and seven had moderate visual loss. At most recent follow-up, three patients were blind, two had severe visual loss and six had moderate visual loss. Of the three blind patients at follow-up, one was blind at screening, one had moderate visual loss, and one had a VA of 0.18 LogMAR.

Visual acuities at screening, first HES appointment, and final follow-up are shown in Figure 3 for those patients with a definitive HES diagnosis of active PDR. Mean HES followup was 4.8 months (range: 0-13 months). Delay in referral greater than 4 weeks did not significantly predict the likelihood of vision loss in all patients referred $\left(\chi^{2}, P=0.49\right)$ or in just those patients with a definitive HES diagnosis of active PDR $\left(\chi^{2}, P=1.00\right)$.

Over the course of the study, 23 patients with active PDR lost at least 0.1 LogMAR VA in at least one of their eyes. Delay in presentation was thought to have led directly to VA loss in three of these patients: two patients developed vitreous haemorrhages and one developed rubeotic glaucoma. In both patients with vitreous haemorrhages, the VA improved to better than referral VA during the course of the study. One patient required vitrectomy and the other spontaneously resolved. Earlier outpatient appointments may have reduced the time course and degree of vision loss in these patients.

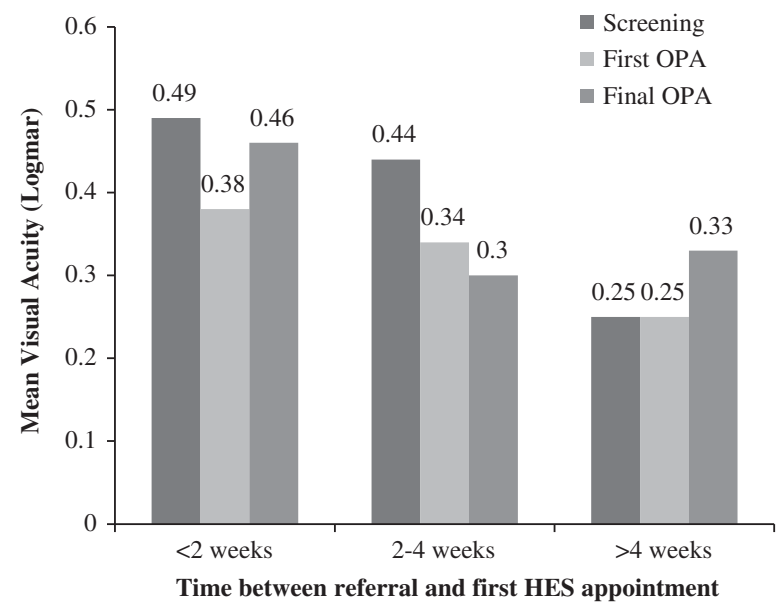

Figure 3 Mean visual acuity changes with time in active PDR patients according to referral time.
Higher HbA1c did not predict increased chance of active PDR (two-sample $T$-test, $P=0.77$ ). It also did not predict increased likelihood of losing vision at some time during the study (two-sample $T$-test, $P=0.57$ ). Mean $\mathrm{HbA} 1 \mathrm{c}$ at first HES appointment was 9.98\% ( $n=37$, SD 2.7) and at final follow-up it was $9.34 \%(n=40$, SD 2.17).

Only 37 of all the patients in the study were new to $\mathrm{MEH}$. The new patients were no more likely to have active PDR $\left(\chi^{2}, P=0.18\right)$ than the others. They were also no more likely to lose vision during the study period $\left(\chi^{2}, P=0.48\right)$.

\section{Discussion}

At the time of this study, 31\% of patients referred to $\mathrm{MEH}$ with a screening programme diagnosis of PDR were seen within 2 weeks. This did not meet the standard of $60 \%$ set by the NHS DESP. Within 4 weeks, $67 \%$ of patients were seen, closer to the $80 \%$ target. The data from nonattendees suggest that if all of the patients had attended their first offered hospital appointment, the figures for 2 weeks and 4 weeks would have been $33 \%$ and $78 \%$, respectively.

Since the time of this study, MEH has significantly changed the clinical pathways and infrastructure and administration of DESP referrals to improve the availability of subspecialty clinic appointments for R3 patients. Consequently, MEH performance has significantly improved, being currently above the national average: $88.7 \%$ of R3a patients seen within 4 weeks (national average: 74.7\%; 2014-2015 Q3 data).

This study shows there may be minimal short-term visual consequence in several weeks of delayed referral for many patients with a diagnosis of R3. This should be reassuring for the clinical teams who work hard to improve attendance at appointments and reduce anxiety in patients with a new diagnosis of R3. Similar findings were suggested in a recent study modelling progression of disease states in diabetic retinopathy using Markov chain predictions compared with a real population. ${ }^{4}$ This study suggested that patients stay for the longest period in the PDR state (nearly 8 years) before transitioning into blindness.

However, the national guidance remains important. This is due to the occasional patient at very high risk of vision loss and the many gains for the patients in terms of time to properly assess medical and ocular conditions and counsel and consent them for treatment where necessary. This group of patients are more often socially deprived, with an increased likelihood of other medical conditions, and they often have a very poor attendance history. ${ }^{5}$ Screening photographs cannot be used to predict hospital eye service attendance record, underlying medical conditions, or response to treatments. Therefore, we believe that prompt referral remains very important. 
Given the importance of the targets, this study has illustrated several ways in which they may be better met.

First, inappropriate referrals to the HES should be reduced. In $53 \%$ of eyes, the hospital grade of diabetic retinopathy differed from the screening programme diagnosis. This may well be because of different examination techniques in the two services, each with their own pros and cons. Disagreements between grading using retinal photography in the DESP and biomicroscopy in the HES are well known, with biomicroscopy seemingly less accurate. ${ }^{6} \mathrm{New}$ technologies, such as wide-field imaging and OCT, being introduced routinely into HES, are improving accuracy of diagnoses. ${ }^{7}$ Wide-field imaging detects more new vessels in more patients than the standard two $45^{\circ}$ images used by the DESP. ${ }^{8}$ These differences in diagnosis continue to challenge both graders and ophthalmologists, but can be resolved with a better understanding of the processes in each service and with better communication between them.

Second, unnecessary referrals need to be reduced. During the course of this study, 47 patients were referred from the screening programme urgently but were already under the care of $\mathrm{MEH}$. There needs to be better communication between the HES and the DESP and better communication with patients regarding the roles of the DESP. At MEH, failsafe officers now actively triage new referrals for patients currently under the care of MEH and inform the screening programme. This study reflects a time period before the introduction of the new common pathway that now differentiates between active PDR (R3a) and stable disease (R3s). This has also recently led to an improvement in this regard.

A weakness of this study is that the influence of macular disease on VA in PDR patients was not analysed because of the small numbers involved. This could be an area for future study. This study did not look specifically at the ratio of high-risk PDR to low-risk PDR and the effect that the type of PDR had on VA loss. An understanding of whether this ratio is changing with time in different locations could be an area for future study for future planning of referral times.

In conclusion, this study provides important evidence that a delay in seeing patients referred from DESP with a diagnosis of R3 may not significantly affect visual outcome in the short term. In the future, as diagnostic accuracy within the DESP improves with the introduction of new technology such as OCT and wide-field imaging, it may well be that future studies demonstrate a more significant impact of delay in referral on vision.

\section{Summary}

What was known before

- National guidance is that $60 \%$ of R3 grade (PDR) patients referred from the DESP should receive a HES consultation within 2 weeks and $80 \%$ should receive a consultation within 4 weeks.

- Patients are known to stay in the PDR state for long periods before transitioning to blindness.

- Patients with PDR are more likely to be socially deprived with multiple other medical conditions.

What this study adds

- There may be little short-term visual consequence in several weeks of delayed referral for many patients with a diagnosis of R3.

- National guidance regarding targets remains important given the vulnerability of this group of patients and the occasional patient at very high risk of visual loss.

- There are several ways by which hospital eye services can meet targets in a better manner, some of which Moorfields Eye Hospital has successfully employed.

\section{Conflict of interest}

The authors declare no conflict of interest.

\section{References}

1 NHS Diabetic Eye Screening Programme. Available at http:/ / diabeticeye.screening.nhs.uk/. Accessed 2 January 2015.

2 Grading diabetic retinopathy from stereoscopic color fundus photographs-an extension of the modified Airlie House classification. ETDRS report number 10. Early Treatment Diabetic Retinopathy Study Research Group. Ophthalmology 1991; 98(5 Suppl): 786-806.

3 Early photocoagulation for diabetic retinopathy. ETDRS Report Number 9. Early Treatment Diabetic Retinopathy Study Research Group. Ophthalmology 1991; 98: 766-785.

4 Srikanth P. Using Markov chains to predict the natural progression of diabetic retinopathy. Int J Ophthalmol 2015; 8(1): 132-137.

5 Lane M, Mathewson PA, Sharma HE, Palmer H, Shah P, Nightingale $\mathrm{P}$ et al. Social deprivation as a risk factor for late presentation of proliferative diabetic retinopathy. Clin Ophthalmol 2015; 9: 347-352.

6 Sallam A, Scanlon PH, Stratton IM, Jones V, Martin CN, Brelen $\mathrm{M}$ et al. Agreement and reasons for disagreement between photographic and hospital biomicroscopy grading of diabetic retinopathy. Diabet Med 2011; 28(6): 741-746.

7 Manjunath V, Papastavrou V, Steel DHW, Menon G, Taylor R, Peto $\mathrm{T}$ et al. Wide-field imaging and OCT vs clinical evaluation of patients referred from diabetic retinopathy screening. Eye 2015; 29: 416-423.

8 Talks J, Manjunath V, Steel DHW, Peto T, Taylor R. New vessels detected on wide-field imaging compared to two-field and seven-field imaging: implications for diabetic retinopathy screening image analysis. Br J Ophthalmol 2015; 99(12): 1606-1609. 